\title{
Determination of Stress Intensity Factors in Low Pressure Turbine Rotor Discs
}

\author{
Ivana Vasovic, ${ }^{1}$ Stevan Maksimovic, ${ }^{2}$ Katarina Maksimovic, ${ }^{3}$ \\ Slobodan Stupar, ${ }^{4}$ Gordana Bakic, ${ }^{4}$ and Mirko Maksimovic ${ }^{5}$ \\ ${ }^{1}$ Institute Gosa, Milana Rakica 35, Belgrade, Serbia \\ ${ }^{2}$ Military Technical Institute, Ratka Resanovica 1, Belgrade, Serbia \\ ${ }^{3}$ City of Belgrade Secretariat for Communal and Housing Affairs Office of Water Management, Kraljice Marije 1/XIII-XIV, \\ 11000 Belgrade, Serbia \\ ${ }^{4}$ Faculty of Mechanical Engineering, University of Belgrade, Kraljice Marije 16, Belgrade, Serbia \\ ${ }^{5}$ Belgrade Waterworks and Sewerage, Kneza Miloša 27, Belgrade, Serbia
}

Correspondence should be addressed to Ivana Vasovic; ivanavvasovic@gmail.com

Received 3 October 2013; Revised 13 January 2014; Accepted 16 January 2014; Published 1 June 2014

Academic Editor: Marek Lefik

Copyright (C) 2014 Ivana Vasovic et al. This is an open access article distributed under the Creative Commons Attribution License, which permits unrestricted use, distribution, and reproduction in any medium, provided the original work is properly cited.

\begin{abstract}
An attention in this paper is focused on the stress analysis and the determination of fracture mechanics parameters in low pressure (LP) turbine rotor discs and on developing analytic expressions for stress intensity factors at the critical location of LP steam turbine disc. Critical locations such as keyway and dovetail area experienced stress concentration leading to crack initiation. Major concerns for the power industry are determining the critical locations with one side and fracture mechanics parameters with the other side. For determination of the critical locations in LP turbine rotor disc conventional finite elements are used here. For this initial crack length and during crack growth it is necessary to determine SIFs. In fatigue crack growth process it is necessary to have analytic formulas for the stress intensity factor. To determine analytic formula for stress intensity factor (SIF) of cracked turbine rotor disc special singular finite elements are used. Using discrete values of SIFs which correspond to various crack lengths analytic formula of SIF in polynomial forms is derived here. For determination of SIF in this paper, combined $J$-integral approach and singular finite elements are used. The interaction of mechanical and thermal effects was correlated in terms of the fracture toughness.
\end{abstract}

\section{Introduction}

Stress intensity factors are very powerful parameters for predicting the loads and crack length at which fracture can occur. The joint between the turbine blade and the disc usually represents the most critical area from the point of view of the static and fatigue approaches. The loads associated with these regions are mainly the centrifugal forces and thermal stresses. Disks are the most strained and responsible steam turbine elements. They contain technological and structural stress concentrators in which damages are eventually accumulated. In low pressure (LP) steam turbine, rotating discs are simultaneously subjected to mechanical and moderate thermal load [1-3]. A disc is loaded under internal pressure due to shrink fit on a shaft. Thus, additional blade effects may be taken into consideration and modeled by an external tensile load at the outer radius of the disc when the disc rotates with significant angular velocity. Material behavior is temperature dependant and changes in material properties throughout the disc should be considered. In order to attain a certain and reliable analysis, solution should consider changes in material specification caused by temperature. Therefore, engineers have strong interests in monitoring and analyzing of rotating components in jet engines to improve safety and to lower maintenance cost. To prevent catastrophic failure of the engine, they have developed different techniques to analyze structures. The engineering field of fracture mechanics was established to develop a basic understanding of such problems. Today, fracture mechanics is not only of academic interest, but also 
plays an increasingly important role in structural design [4-6]. Recently, a more stringent safety criterion, assuming a preexisting flaw in critical component, has been adopted to assess service life of aircraft [7-10]. This emphasizes the significance of fracture mechanics as a tool for analysis. The concepts of linear elastic fracture mechanics which lead to the strain fracture toughness property, $K_{I C}$, have already been used in engineering applications. Mechanical loading is not the only factor considered in the design of structures or structural components. Other possible situations maybe operational environments, such as temperature, have to be considered. In the operation of gas turbine engines, for example thermal stresses can be as high as, or higher than, the centrifugal stresses. The worst condition of the combinations of thermal, centrifugal, and gas bending stresses at elevated temperatures results in high local stresses which can lead to cracking of the LP turbine blades and rotor disks. Thus, thermal effects should not be ignored. A special attention in this paper is focused to develop analytic expressions for stress intensity factors at low pressure steam turbine disc. Finite element method (FEM) is used to determine critical location, zone of the stress concentration at low pressure steam turbine disc, in which rotor blades are connected with rotation disc. At critical location of rotation disc initial crack lengths are assumed. For various crack lengths at LP steam turbine disc stress intensity factors are determined under thermomechanical loads. Using these discrete values of SIFs here are derived analytic formulas for SIF, $K_{I}$, at cracked LP disc. Analytic formula of the stress intensity factor information is valuable in the prediction of service lives of turbine discs. Crack growth behavior is a major issue in a variety of rotating components for which analytic formula of SIF is necessary.

The primary objective of this research is to establish complete budgetary procedures related to the design of structural elements of low pressure turbine disc in the presence of initial crack. This approach to design in the presence of initial cracks is known as the "damage tolerance approach." With this approach, where the initial damage is at the critical locations of the turbines, the risk of unforeseen catastrophic failures during operation is reduced to a minimum.

Low Pressure Turbine. Large scale steam turbines employed for the generation of electricity are expected to operate for working lives of 30 years and beyond. A harsh combination of service temperature and environment dictates that the most severely stressed components within the low pressure turbine will need to be formed from what are generally classified as high performance materials.

In light of such experiences, the present study was commissioned to investigate the fatigue response of a common LP blade material, the X12CrNiMoVNb steel. An improved understanding of the mechanical behaviour under variable loading conditions is deemed.

Failures occur from time to time in power plants, as they do in other engineering structures. However, they are not always examined closely to identify the causes. Similarly, when the failures are observed from time to time, repairs are often made without any careful analysis being undertaken.
Most blade failure investigations end with a metallurgical report. A metallurgical examination of the blade establishes whether the failure was due to substandard material or due to the presence of flaws, machine marks, or corrosion pits that created local stress raisers not accounted for in the allowable stress limits of the blade design. A fracture surface of the blade can suggest that fatigue is a more probable failure mechanism. But the correction of a blade problem requires more than positive identification of the mechanisms involved [3].

Finite element analysis needs to be performed because of complex geometry and boundary conditions, such as the blade. So, finite element analysis is used to calculate the stresses and modal shapes of the models. Previous researches have shown that the low pressure blades of a steam turbine are generally found to be more susceptible to failure than intermediate pressure and high pressure blades [11]. The most common failure mechanisms, which occur within the low pressure blade, are normally those associated with either sympathetic or forced vibrations, those caused by transient operating conditions, and those that occur as the result of the transported and accumulated corrosive ions in working fluid $[3,12-17]$. Blades and low pressures of steam turbine discs are critical components in power plants that convert the available energy in steam into mechanical energy [1].

\section{Strength Analysis with respect to Fracture Mechanics}

The objective of the analytical work is to provide the relationship between the stress intensity factor and the various mechanical and thermal load conditions. Residual fatigue life of cracked rotation components is generally based upon crack growth consideration. Fracture mechanics is the mathematical tool that is employed. It provides the concepts and equations used to determine how cracks grow and their effect on the strength of structure. From an initial crack length $a_{o}$ one must determine critical flow size $a_{c}$ for the fast fracture damage tolerance approach:

$$
\begin{gathered}
a_{o} \longrightarrow a_{c}, \\
K_{I} \longrightarrow K_{I C},
\end{gathered}
$$

where $K_{I}$ is the applied tensile mode, $I$ is the stress intensity factor, and $K_{I C}$ is the strain fracture toughness of material.

In fracture mechanics, we try to correlate analytical predictions of crack growth and failure with experimental results. The analytical predictions made by calculating fracture parameters such as stress intensity factors in the crack region, can be used to estimate crack growth rate. Although several stress intensity factor handbooks $[5,18]$ have published the available solutions, those solutions are not always adequate for particular engineering applications. This is especially true for cracks subjected to nonuniform stress fields near notch or thermal stresses $[6,19]$. Stress intensity factor calculation is an important issue for numerical analysis of fracture problems and there exist many approaches, for 
instance, the extrapolation techniques, $J$-integral approach $[20,21]$, the virtual crack extension (VCE) technique, and so forth. Amongst these methods, a displacement extrapolation method sometimes called crack opening displacement correlation technique used specially with the quarter-point singular finite elements (QPE) [21] is most widely used due to its high accuracy and simplicity.

Typical fracture parameters of interest are stress intensity factors $\left(K_{I}, K_{I I}\right.$, and $\left.K_{I I I}\right)$, which correspond to three basic modes of fracture $J$-integral [22], a path-independent line integral that measures the strength of the singular stresses and strains near a crack tip, and energy release rate $(G)$, which represents the amount of work associated with a crack opening or closure.

Firstly, linear elastic or elastic-plastic static analysis has to be performed and then we use postprocessing commands to calculate fracture parameters.

Finite element method (FEM) is the most widely used technique for evaluating stress intensity factor (SIF). The most important region in modeling the fracture region is the region around the crack tip. While the domain is meshed, we are using crack tip singular finite elements with nodal singularity $[6,20]$. Those elements exhibit the $r^{-1 / 2}$ singularity both on the boundary of the element and in the interior. Displacement correlation was employed to determine stress intensity factors. Maksimović et al. [9] employed hybrid finite elements with a square root surrounding the crack tip and regular elements in the rest of the domain to determine stress intensity factors.

One main objective of this paper lies in developing a complete computation procedure for the strength analysis of cracked structural components, based on combining $J$ integral approach and singular finite elements.

2.1. Determination of the Stress Intensity Factors. Once a finite element solution has been obtained, the values of the stress intensity factor can be extracted from it. Determination of the stress intensity factors of cracked structural components based on finite element analysis (FEA) and linear elastic fracture mechanics (LEFM) can be done. In this paper the stress analysis of LP turbine disc with initial crack is considered using the finite element program ANSYS [23]. The computer program evaluates the stress intensity factors (SIFs) on the crack tip from the inner side of hole in turbine disc. The program requires one global model of disc with or without modeled crack and one detailed submodel of investigated crack. The program makes it easy to build submodel from an already existing global model. The global model can preferably be meshed with tetrahedral elements, which is one of the strongest specified demands.

The crack submodel is very easy to create in an already existing globally meshed model. In this procedure the traditional submodeling technique will be used, where the displacement field of the global model is applied as boundary conditions on the submodel. The global model must contain the actual crack length; otherwise the SIF will be incorrect due to reduced stiffness of the submodel.

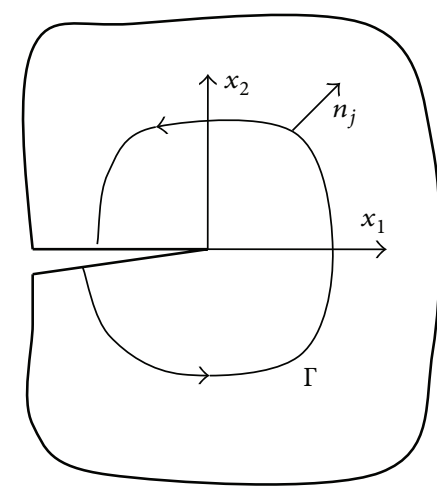

FIGURE 1: Arbitrary contour and coordinate configuration.

The global model has tetrahedral elements around the crack tip making it impossible to achieve the SIF. To solve this problem a small local submodel with 20 -node hexahedral elements with singular elements is generated around the crack tip.

SIFs are obtained from the FEM and $J$-integral methods available in ANSYS software code [23]. The stress intensity factor was evaluated using the following equivalent relationship:

$$
K=\sqrt{\frac{E J}{1-v^{2}}},
$$

where $E$ is Young's modulus, $v$ is Poisson's ratio, and $J$ integral is evaluated using ANSYS. The path-independent $J$ line integral which was proposed by Rice [22] is defined as

$$
J=\int_{\Gamma}\left(W d x_{2}-\sigma_{i j} \frac{\partial u_{i}}{\partial x_{1}} n_{j} d S\right)
$$

where $W$ is the elastic strain energy density, $\Gamma$ is any contour about the crack tip shown in Figure $1, T_{i}$ and $u_{i}$ are the traction and displacement components along the contour, $S$ is arc length along the contour, and $x_{1}$ and $x_{2}$ are the local coordinates such that $x_{1}$ is along the crack.

Generally, the stress intensity factors are additive and provide different loading conditions that induce the same mode of crack extension. Hence, the fracture condition of the interaction of mechanical and thermal loads can be

$$
K_{I}=\left(K_{I}\right)_{M}+\left(K_{I}\right)_{T}
$$

when $K_{I}=K_{I C}$, in which $\left(K_{I}\right)_{M}$ is the stress intensity factor due to mechanical load and $\left(K_{I}\right)_{T}$ is the stress intensity factor due to temperature effect.

Unfortunately, the $J$-integral is restricted to twodimensional bodies with external loading. The $J$-integral is path dependent for cases which include residual, inertial, or thermal stress terms or loadings along the crack face. It cannot be used for three-dimensional structures of nonhomogeneous materials in the direction of crack advance [24]. Some efforts have been made to modify the expression 


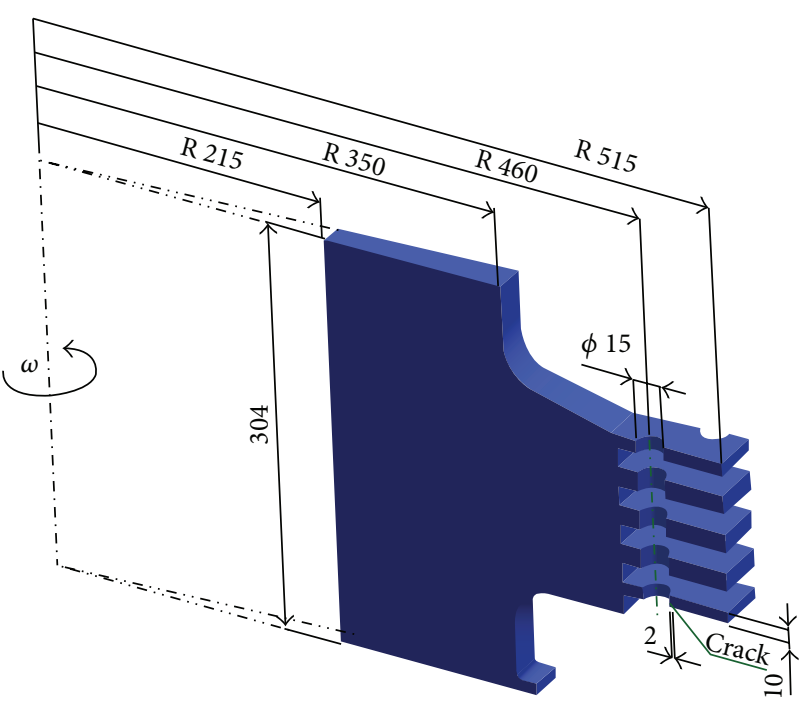

FIGURE 2: Geometry properties of part of low pressure turbine disc with initial crack.

of $J$-integral. Wilson and Yu's [25] integral is valid for use in two-dimensional thermal cases. Blackburn et al.'s modified integral [26] is applicable to three-dimensional cases. In this study the modified integral $J^{*}[26]$ was used as

$$
\begin{gathered}
J^{*}=\int_{\Gamma}\left(W^{*} d x_{2}-\sigma_{i j} \frac{\partial u_{i}}{\partial x_{1}} n_{j} d S\right)+\frac{E \alpha}{1-2 \nu} \int \varepsilon_{i i} \frac{\partial \theta}{\partial x_{1}} d A, \\
W^{*}=W-\frac{E \alpha \theta}{2(1-2 \nu)} \varepsilon_{i i},
\end{gathered}
$$

in which

$$
\begin{gathered}
\sigma_{i j}=\lambda \varepsilon_{i i} \delta_{i j}+2 \mu \varepsilon_{i j}-\frac{E \alpha}{1-2 \nu} \theta \delta_{i j}, \\
W=\frac{1}{2} \sigma_{i j} \varepsilon_{i j},
\end{gathered}
$$

where $\mu$ and $\lambda$ are Lame's constants, $\theta$ is the temperature, and $\alpha$ is the coefficient of thermal expansion. If only mode $I$ loading is considered, then (5) reduces to

$$
J^{*}=\frac{\left(1-\nu^{2}\right) K_{I}^{2}}{E} .
$$

Accordingly, $J$-integral is modified to include the elasticplastic and thermal effects whilst maintaining the independence of the trajectory along which integral is determined $[27,28]$.

Combining FEM with modified $J^{*}$-integral approach to analyze thermomechanical problems with respect to fracture mechanics is considered in the articles [25-31].

\section{Numerical Analysis and Validation}

Attention in this investigation is focused on determination of the stress intensity factors in the cracked turbine discs.

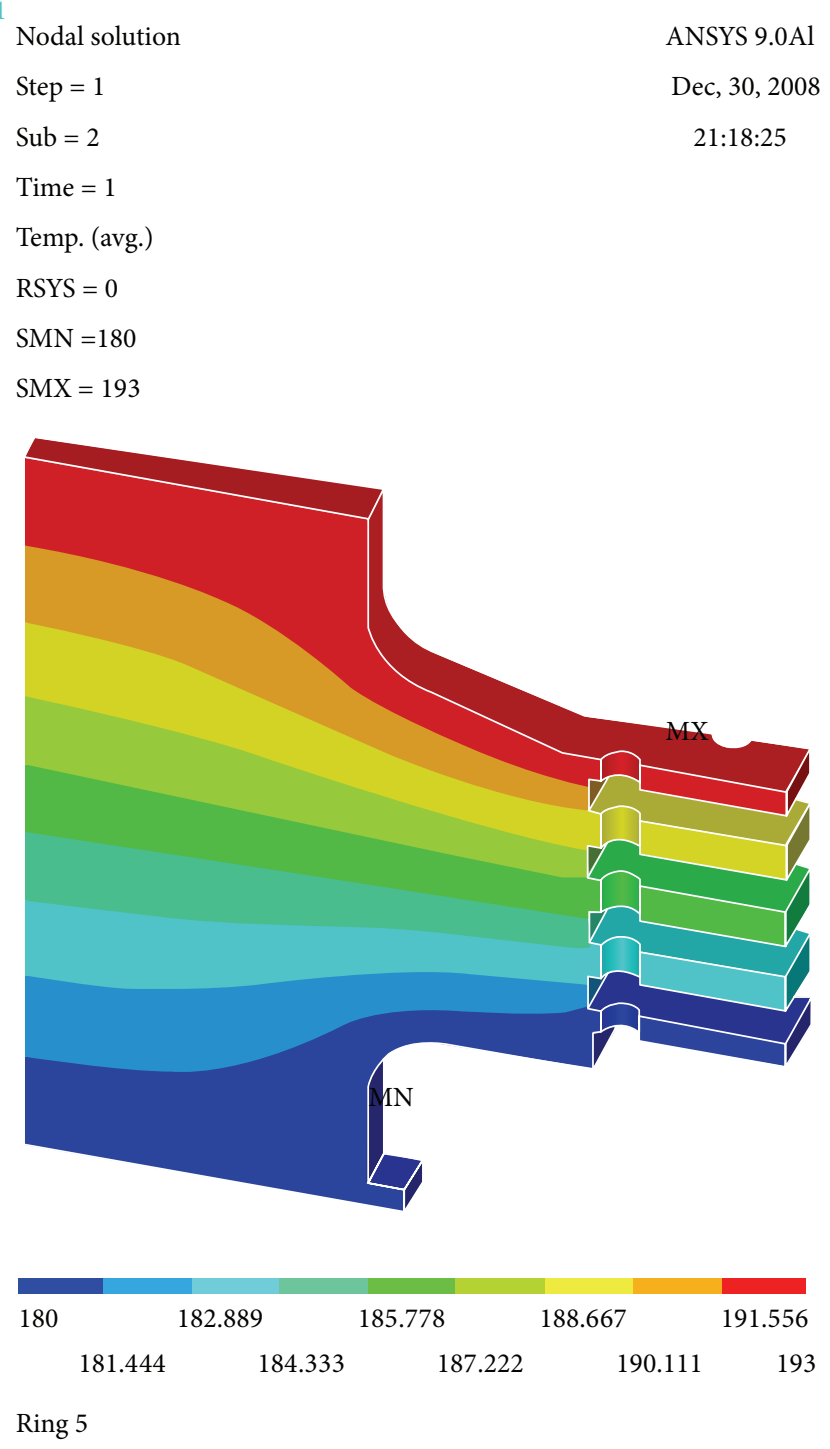

FIgURE 3: Temperature distributions at part of a low pressure turbine disc.

In determining fracture mechanical parameters, primarily stress intensity factors, we used finite element method and $J$-integral approach. On basis of external load conditions, the temperature on the upper surface of rotor disc is $193^{\circ} \mathrm{C}$ and on the lower surface is $180^{\circ} \mathrm{C}$ is using SOLID70 type finite element that is incorporated in ANSYS software code [23]. Geometry properties of the part turbine disc including location of initial crack length are shown in Figure 2. Turbine disc is under combined mechanical load due to rotation speed and due to temperature distributions. Temperature distributions at part of a low pressure turbine disc are shown in Figure 3.

In stress analysis, disc was modeled with SOLID95 type elements consisting of twenty-node solid elements (Figures 4 and 5). These elements are suitable for modeling area around crack tip and correctly describe singular stress and strain fields (Figure 6). Calculated stress values around crack 


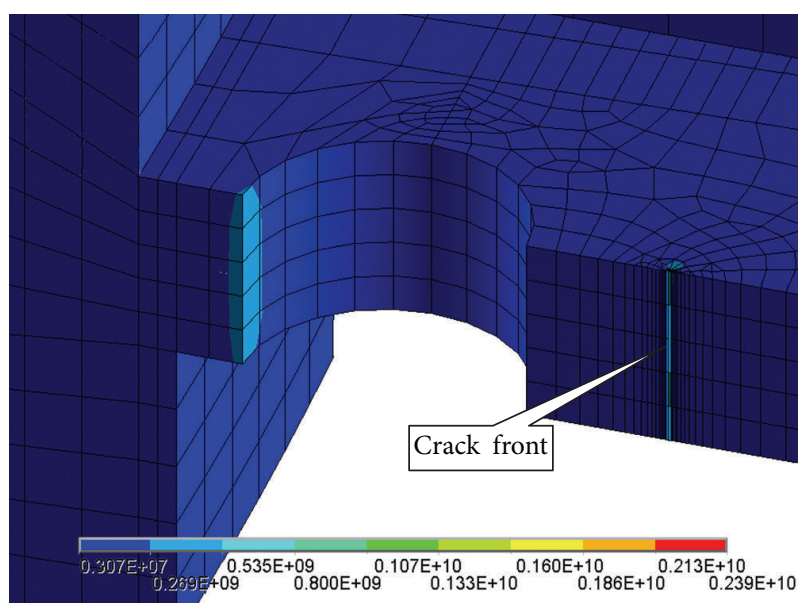

Figure 4: Von Misses stresses from thermal and mechanical loads $(n=3000 \mathrm{o} / \mathrm{min})$ in elements, crack length $a=0.001 \mathrm{~m}$.

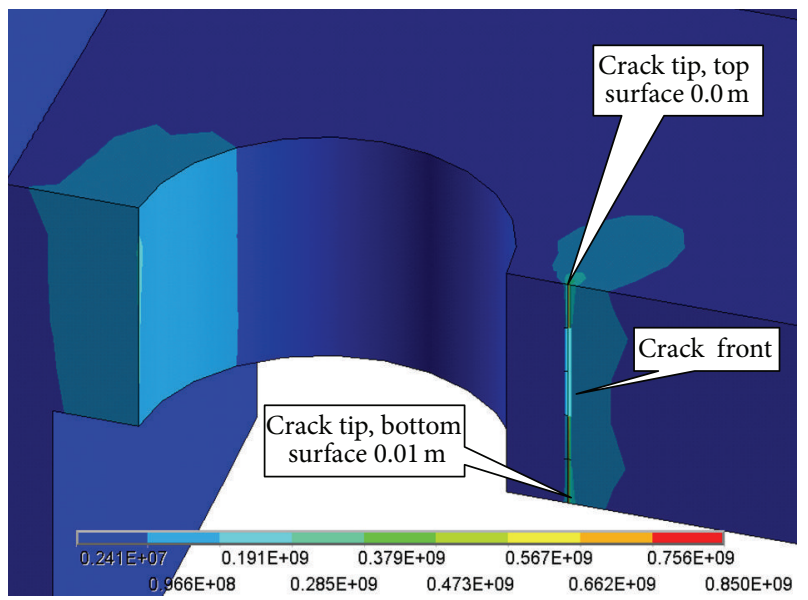

Figure 5: Von Misses stresses in submodel from thermal and mechanical loads ( $n=3300 \mathrm{o} / \mathrm{min}$ ), crack length $a=0.0025 \mathrm{~m}$.

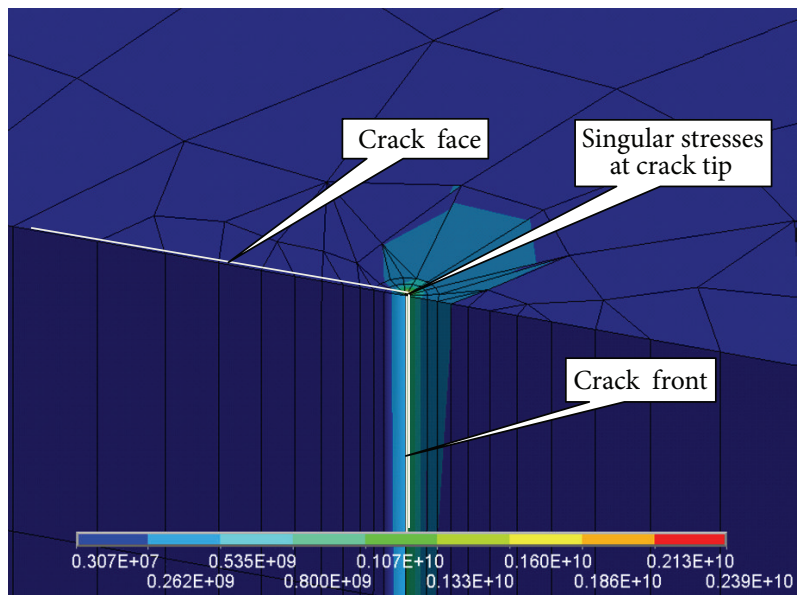

Figure 6: Von Misses stresses in submodel from thermal and mechanical loads $(n=3000 \mathrm{o} / \mathrm{min})$ in elements, crack length $a=$ $0.001 \mathrm{~m}$.
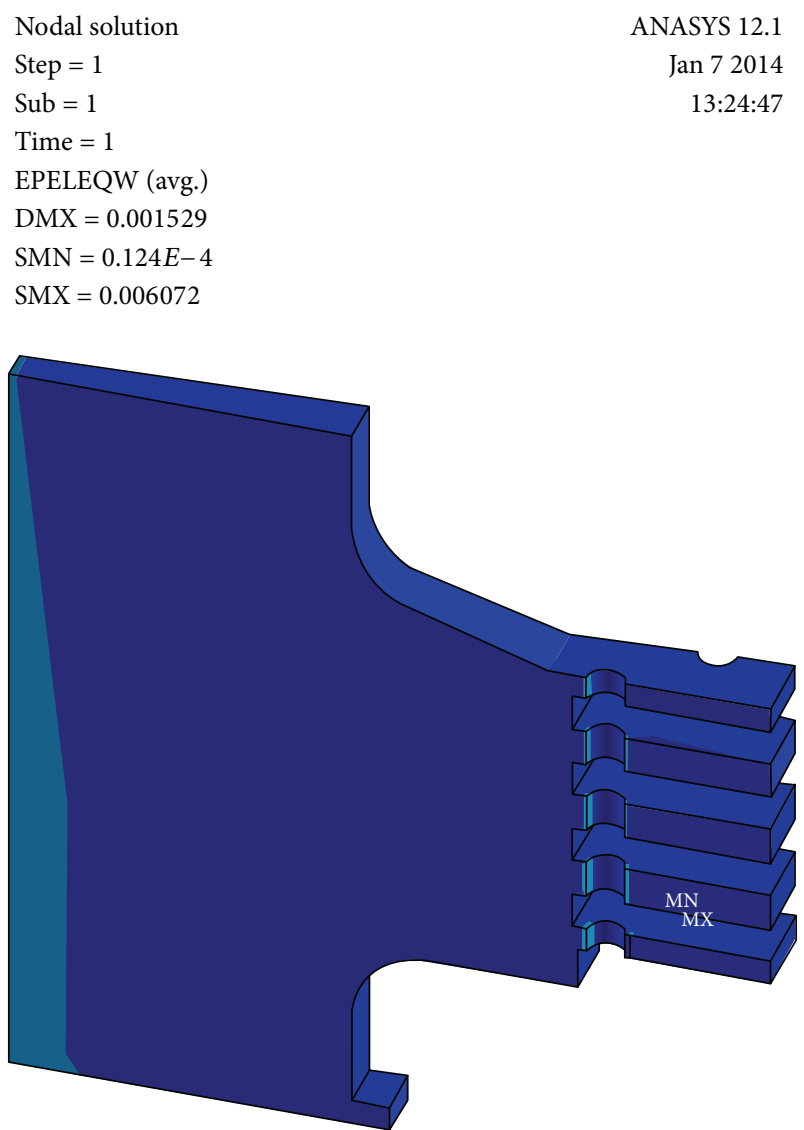

$0.002706 \quad 0.004052$

0.005399

$$
\begin{array}{lllll}
0.686 E-03 & 0.002032 & 0.003379 & 0.004726 & 0.006072
\end{array}
$$

Turbine disc $a=2.0 \mathrm{~mm}$

FIGURE 7: Elastic deformation in turbine disc $(n=3000 \mathrm{o} / \mathrm{min})$ in elements, crack length $a=2 \mathrm{~mm}$.

tips were used to determine stress intensity factors using $J$ integral approach.

In Figures 7 and 8 elastic deformations and displacements in turbine discs are illustrated for the crack length $2 \mathrm{~mm}$. Von Misses stress distributions in cracked turbine disc, for initial crack $2 \mathrm{~mm}$, are shown in Figure 9.

Values of stress intensity factors for different crack sizes and loads are shown in Table 1 and Figure 10. Disks were made of $0.35 \mathrm{C} 0.65 \mathrm{Mn} 0.9 \mathrm{Cr} 0.3 \mathrm{Mo}$ steel grade steel (34HN3M steel according to GOST). Material properties for the disk of the turbine are as follows: density $\rho=7820 \mathrm{~kg} / \mathrm{m}^{3}$, Young modulus $E=186 \mathrm{GPa}$, Poisson coefficient $\nu=0.3$, coefficient of thermal expansion $\alpha=13.32 \mu \mathrm{m} / \mathrm{m}^{\circ} \mathrm{C}$, thermal conductivity $\lambda=36 \mathrm{~W} / \mathrm{m}^{\circ} \mathrm{C}$, and $K_{I C}=120.00 \mathrm{MPa}(\mathrm{m})^{1 / 2}$.

Previous finite element results can be used to determine analytic expressions for stress intensity factors. These analytic expressions for stress intensity factors can be used for crack growth analyses and residual life estimations of cracked disc. 

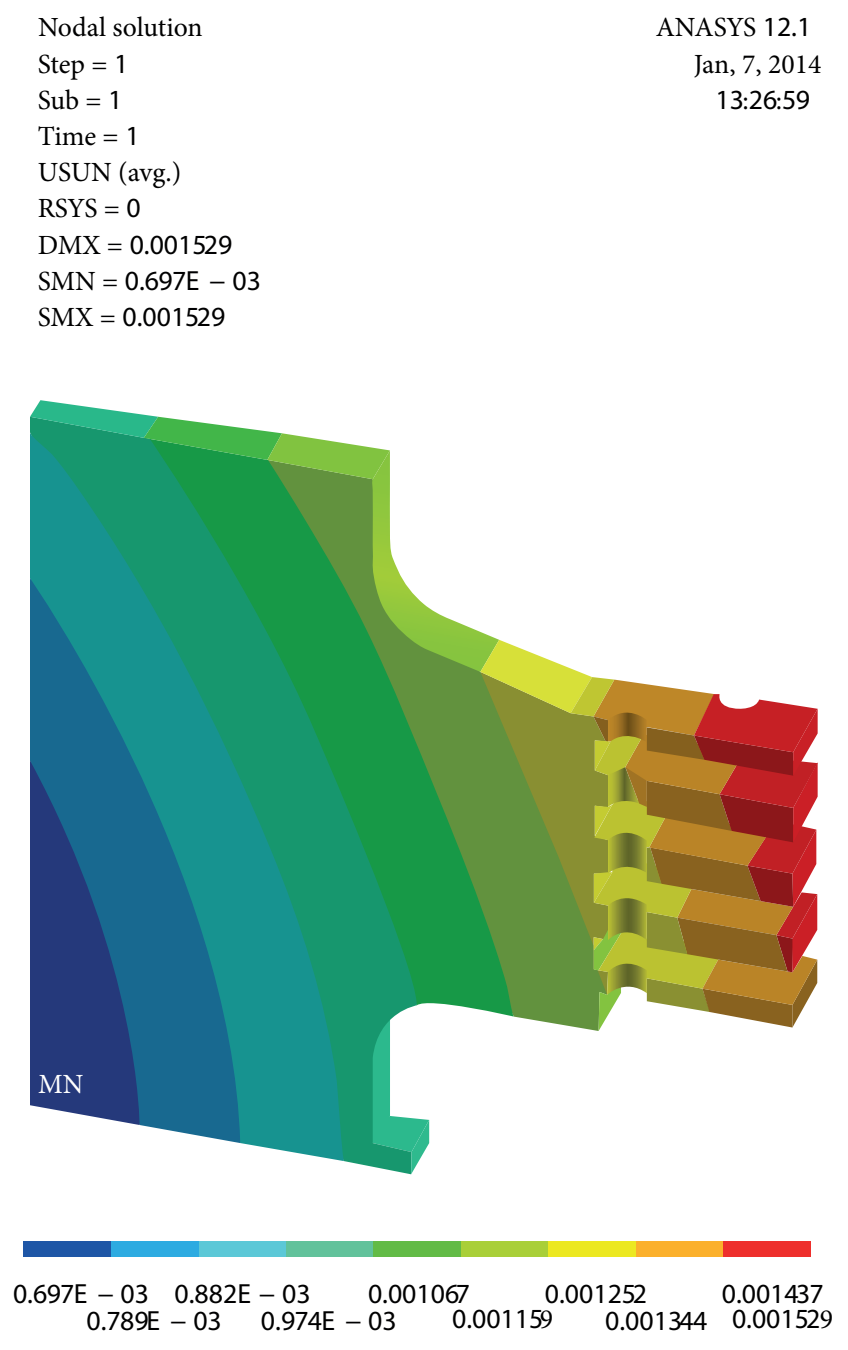

Turbine disc $\mathrm{a}=2.0 \mathrm{~mm}$

FIGURE 8: Displacement in turbine disc $(n=3000 \mathrm{o} / \mathrm{min})$ in elements, crack length $a=2 \mathrm{~mm}$.

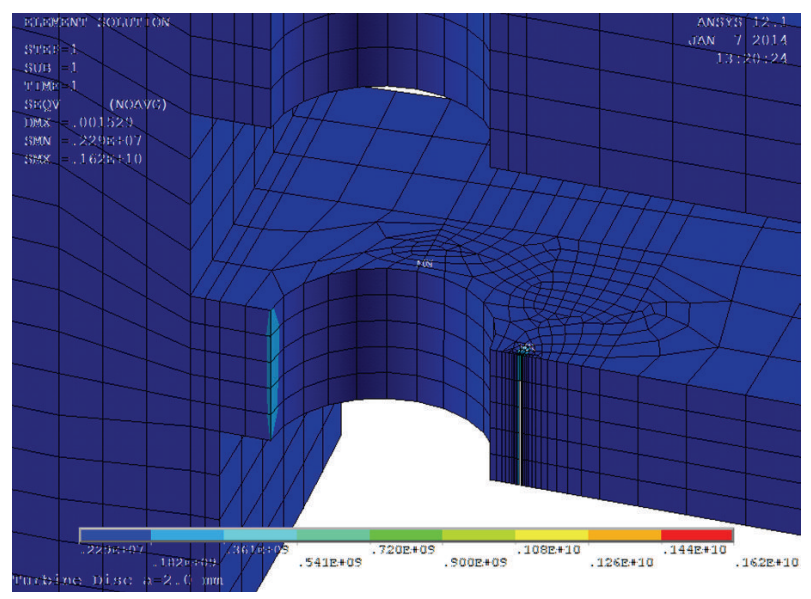

FIgURE 9: Von Misses stresses in turbine discs, $(n=3000 \mathrm{o} / \mathrm{min})$ in elements, crack length $a=2 \mathrm{~mm}$.

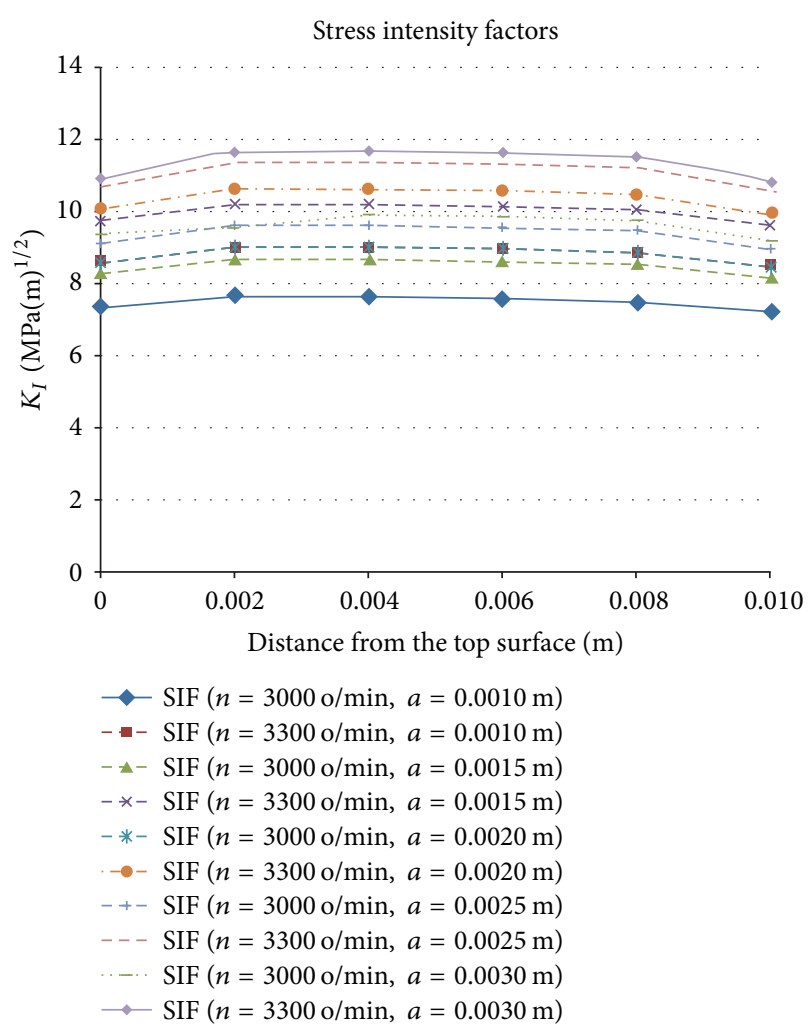

FIGURE 10: Stress intensity factors for different crack lengths and rotational speed.

By using discrete values of stress intensity factors for different crack lengths as shown in Table 1, analytic expressions were established for different load conditions.

The values of stress intensity factors can be found by solving (9) and (10) for different crack lengths, $a$, on the top surface of disc and rotational speed $(n=3000 \mathrm{o} / \mathrm{min}$ and $n=3300 \mathrm{o} / \mathrm{min}$, Figure 11), respectively:

$$
\begin{aligned}
& K_{I 1}=237466666.67 a^{3}-1781314.29 a^{2}+5172.89 a+4.00 \\
& K_{I 2}=240466666.67 a^{3}-1854314.29 a^{2}+5616.44 a+5.02
\end{aligned}
$$

in which $K_{I}\left[\mathrm{MPa}(\mathrm{m})^{1 / 2}\right]$, a [m] is the crack length.

Using previously derived analytic formulas for the stress intensity factors $K_{I}$ we can to control situation in which $K_{I}$ will be equal to its critical value $K_{I C}$. In accordance to computation results, under thermomechanical loads the maximum value of the SIF is $K_{I}=11.65 \mathrm{MPa}(\mathrm{m})^{1 / 2}$, Table 1 , and its fracture toughness is $K_{I C}=120.0 \mathrm{MPa}(\mathrm{m})^{1 / 2}$. In this investigation derived analytic relations (9) and (10) for the stress intensity factors are used for determination of the reserve factor $(\mathrm{RF})$ with respect to "static" fracture mechanics that is defined as

$$
\mathrm{RF}=\frac{K_{I C}}{K_{I}}
$$


TABLE 1: Stress intensity factors for different crack lengths and rotational speed.

\begin{tabular}{|c|c|c|c|c|c|c|c|c|c|}
\hline & & & & \multicolumn{6}{|c|}{$K_{I}\left[\mathrm{MPa}(\mathrm{m})^{1 / 2}\right]$} \\
\hline \multirow{10}{*}{ Crack lengths [m] } & $a=0.0010 \mathrm{~m}$ & \multirow{10}{*}{ Rotational speed $[\mathrm{o} / \mathrm{min}]$} & $n=3000$ & 7.3373 & 7.6519 & 7.6059 & 7.5651 & 7.4727 & 7.2176 \\
\hline & $a=0.0010 \mathrm{~m}$ & & $n=3300$ & 8.6310 & 8.9799 & 8.9979 & 8.9631 & 8.8407 & 8.5053 \\
\hline & $a=0.0015 \mathrm{~m}$ & & $n=3000$ & 8.2728 & 8.6502 & 8.6411 & 8.6006 & 8.5149 & 8.1400 \\
\hline & $a=0.0015 \mathrm{~m}$ & & $n=3300$ & 9.7197 & 10.1864 & 10.1855 & 10.1434 & 10.0165 & 9.5799 \\
\hline & $a=0.0020 \mathrm{~m}$ & & $n=3000$ & 8.5604 & 9.0005 & 8.9857 & 8.9428 & 8.8593 & 8.4211 \\
\hline & $a=0.0020 \mathrm{~m}$ & & $n=3300$ & 10.0724 & 10.6150 & 10.6085 & 10.5644 & 10.4798 & 9.9285 \\
\hline & $a=0.0025 \mathrm{~m}$ & & $n=3000$ & 9.0887 & 9.5932 & 9.5977 & 9.5547 & 9.4731 & 8.9392 \\
\hline & $a=0.0025 \mathrm{~m}$ & & $n=3300$ & 10.6963 & 11.3151 & 11.3304 & 11.2683 & 11.2036 & 10.5392 \\
\hline & $a=0.0030 \mathrm{~m}$ & & $n=3000$ & 9.3531 & 9.5389 & 9.8753 & 9.8345 & 9.7341 & 9.1821 \\
\hline & $a=0.0030 \mathrm{~m}$ & & $n=3300$ & 10.8985 & 11.6519 & 11.6484 & 11.6067 & 11.5023 & 10.8108 \\
\hline \multicolumn{4}{|c|}{ Position of crack tip from the top surface [m] } & 0 & 0.002 & 0.004 & 0.006 & 0.008 & 0.010 \\
\hline
\end{tabular}

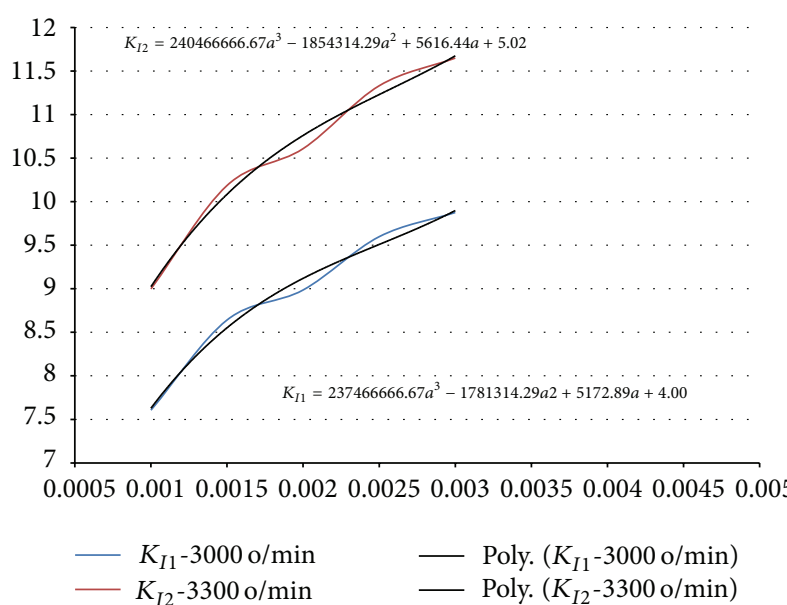

FIGURE 11: Polynomial form of derived stress intensity factors based on discrete values of stress intensity factors.

The relations (9) and (10) can be used too for the crack growth analyses of damaged structural components and residual fatigue lives estimations.

The crack growth of the disc due to fatigue or stress corrosion can be predicted in accordance with the theory of the conventional fracture mechanics. The dependence of the fatigue crack growth rate on the stress intensity factor (9) or (10) can be conveniently represented as follows:

$$
\frac{d a}{d N}=C(\Delta K)^{m}\left[\frac{1-\left(\Delta K_{t h} / \Delta K\right)^{n_{1}}}{1-\left(\Delta K_{\max } / K_{c}\right)}\right]^{n_{3}}
$$

where $d a / d N$ is the fatigue crack growth the rate, $K_{t h}$ is the threshold value of stress intensity factor, $K_{c}$ is the critical value, and $C$ and $n$ are constants depending on the material and environmental conditions.

3.1. Estimating Remaining Life. The remaining life of LP turbine discs for keyway cracking is calculated by the initial crack size $\left(a_{i}\right)$, the critical size $\left(a_{\mathrm{cr}}\right)$, and the crack growth rate $(d a / d t)$ and is expressed by the following relationship:

$$
t_{\text {rem }}=\frac{a_{\mathrm{cr}}-a_{i}}{d a / d t} .
$$

Previous relations (12) and (13) can be used for residual life estimation of low pressure turbine discs.

\section{Conclusions}

This work established the complete computation procedure for derivation of analytic expressions for the stress intensity factors of the complex cracked structural element, such as steam low pressure turbine disc. In order to get high operation availability and to establish a maintenance and spares management strategy capable of developing the life extension of steam turbine suitable assessment of aging damage and residual life estimation for most relevant parts of turbine is essential. This investigation focused on developing analytic expressions for stress intensity factors, at the critical location of LP turbine disc. FEM is used for defining the critical location with respect to fracture mechanics and for the determination of the stress intensity factors. The stress intensity factor information is valuable in the prediction of the service lives of turbine discs. The method, developed in this paper, was applied to compute the stress intensity factors, in domain of linear elastic facture mechanics (LEFM), in plain stress problem. The finite element method has been used, to calculate thermal and mechanical stresses, in a low pressure turbine disc too. These results were used to determinate the stress intensity factors, using $J$-integral approach. In many cases, the $J$-integral can be the easiest means of calculating stress intensity factors. This method is easy to use, when the software supports determination of the contour integral. The results are fairly accurate, even for the coarse meshes because the contours can be chosen to be remote from the near crack tip region. The computed discrete values of stress intensity factors, by finite elements, are used for derivation equations (9) and (10), which present a new analytical expression for the stress intensity factors, where 
value $a$ is the crack length. The derived analytic expressions for the stress intensity factors can be used for the "static" fracture mechanics analysis (11) or residual life estimation of damaged low pressure turbine components using relations (12) and (13).

\section{Conflict of Interests}

The authors declare that there is no conflict of interests regarding the publication of this paper.

\section{Acknowledgments}

This work was financially supported by the Ministry of Science and Technological Developments of Serbia under Projects OI-174001 and TR-35045.

\section{References}

[1] C. Liu and D. D. Macdonald, "Prediction of failures of lowpressure steam turbine disks," Journal of Pressure Vessel Technology, vol. 119, no. 4, pp. 393-400, 1997.

[2] G. R. Jovičič, V. K. Grabulov, S. M. Maksimovič et al., "Residual life estimation of a thermal power plant component-the highpressure turbine housing case," Thermal Science, vol. 13, no. 4, pp. 99-106, 2009.

[3] H.-J. Kim, "Fatigue failure analysis of laststage blade in a low pressure steam turbine," Engineering Failure Analysis, vol. 6, no. 2, pp. 93-100, 1999.

[4] M. Shankar, K. Kumar, and S. L. Ajit Prasad, "T-root blades in a steam turbine rotor: A case study," Engineering Failure Analysis, vol. 17, no. 5, pp. 1205-1212, 2010.

[5] A. G. Evans, "Perspectives on the development of hightoughness ceramics," Journal of the American Society, vol. 73, pp. 187-206, 1990.

[6] R. D. Henshell and K. G. Shaw, "Crack tip finite elements are unnecessary," International Journal for Numerical Methods in Engineering, vol. 9, no. 3, pp. 495-507, 1975.

[7] M. Blažić, K. Maksimović, and Y. Assoul, "Determination of stress intensity factors of of structural elements by surface cracks," in Proceedings of the 3rd Serbian Congress Theoretical and Applied Mechanics, pp. 374-383, Serbian Society of Mechanics, Vlasina Lake, Serbia, July 2011.

[8] S. Boljanović and S. Maksimović, "Fatigue crack growth modeling of attachment lugs," International Journal of Fatigue, vol. 58, pp. 66-74, 2013.

[9] S. Maksimović, S. Posavljak, K. Maksimović, V. Nikolic, and V. Djurkovic, "Total fatigue life estimation of notched structural components using low-cycle fatigue properties," Strain, vol. 47, no. 2, pp. 341-349, 2011.

[10] S. Maksimović, I. Vasović, M. Maksimović, and M. Đurić, "Residual life estimation of damaged structural components using low-cycle fatigue properties," in Proceedings of the $3 \mathrm{rd}$ International Congress of Serbian Society of Mechanics, Vlasina Lake, Serbia, July 2011.

[11] G. Das, S. Ghosh Chowdhury, A. Kumar Ray, S. Kumar Das, and D. Kumar Bhattacharya, "Turbine blade failure in a thermal power plant," Engineering Failure Analysis, vol. 10, no. 1, pp. 8591, 2003.
[12] W. Z. Wang, F.-Z. Xuan, K.-L. Zhu, and S.-T. Tu, "Failure analysis of the final stage blade in steam turbine," Engineering Failure Analysis, vol. 14, no. 4, pp. 632-641, 2007.

[13] L. C. White, Modern Power Station Practice, British Electricity International, Pergamon Press, 1992.

[14] R. Viswanathan, Damage Mechanisms and Life Assessment of High Temperature Components, ASM International, Metals Park, Ohio, USA, 1989.

[15] Y. Zhang, M. Urquidi-MacDonald, G. R. Engelhardt, and D. D. MacDonald, "Development of localized corrosion damage on low pressure turbine disks and blades: I. Passivity," Electrochimica Acta, vol. 69, pp. 1-11, 2012.

[16] Y. Zhang, M. Urquidi-MacDonald, G. R. Engelhardt, and D. D. MacDonald, "Development of localized corrosion damage on low pressure turbine disks and blades: II. Passivity breakdown," Electrochimica Acta, vol. 69, pp. 12-18, 2012.

[17] Y. Zhang, M. Urquidi-MacDonald, G. R. Engelhardt, and D. D. MacDonald, "Development of localized corrosion damage on low pressure turbine disks and blades. III: application of damage function analysis to the prediction of damage," Electrochimica Acta, vol. 69, pp. 19-29, 2012.

[18] J. W. Hutchinson and Z. Suo, "Mixed mode cracking in layered materials," in Advances in Applied Mechanics, J. W. Hutchinson and T. Y. Wu, Eds., vol. 29, pp. 63-191, Academic Press, Orlando, Fla, USA, 1992.

[19] S. T. Lin and R. E. Rowlands, "Thermoelastic stress analysis of orthotropic composites," Experimental Mechanics, vol. 35, no. 3, pp. 257-265, 1995.

[20] R. S. Barsoum, “Triangular quarter-point elements as elastic and perfectly-plastic crack tip elements," International Journal for Numerical Methods in Engineering, vol. 11, pp. 85-98, 1977.

[21] R. S. Barsoum, "On the use of isoparametric finite elements in linear fracture mechanics," International Journal for Numerical Methods in Engineering, vol. 10, no. 1, pp. 25-37, 1976.

[22] J. R. Rice, "A path independent integral and approximate analysis of strain concentration by notches and cracks," Journal of Applied Mechanics, vol. 35, pp. 379-386, 1968.

[23] ANSYS, Finite Element Software Code.

[24] A. R. Zak and M. L. Williams, "Crack point singularities at a bimaterial interface," Journal of Applied Mechanics, vol. 30, pp. 142-143, 1963.

[25] W. K. Wilson and I.-W. Yu, "The use of the J-integral in thermal stress crack problems," International Journal of Fracture, vol. 15, no. 4, pp. 377-387, 1979.

[26] W. S. Blackburn, A. D. Jackson, and T. K. Hellen, "An integral associated with the state of a crack tip in a non-elastic material," International Journal of Fracture, vol. 13, no. 2, pp. 183-199, 1977.

[27] S. Maksimović, "Finite elements in thermoelastic and elastoplastic fracture mechanics," in Proceedings of the 3rd International Conference Held University Held at College, Swansea, pp. 495-504, March 1984.

[28] J. R. Rice, "Elastic fracture mechanics concepts for interfacial cracks," Journal of Applied Mechanics, vol. 55, no. 1, pp. 98-103, 1988.

[29] S. Maksimović, "An investigation of the effect of thermal gradients on fracture," in Proceedings of the 6th International Conference on Fracture (ICF '6), vol. 2, pp. 4-10, Pergamon Press, New Delhi, India, December 1984. 
[30] D. Stamenkovic, Evaluation Fracture Mechanics Parameters of Thermally Loaded Structures, Scientific Technical Review, no. 2, 2008.

[31] D. Stamenkovic, "Determination of fracture mechanics parameters using FEM and J-integral approach, finite element simulation of the high risk constructions," in Proceedings of the 2nd WSEAS International Conference on Applied and Theoretical Mechanics (MECHANICS '06), D. Mijuca and S. Maksimović, Eds., Venice, Italy, 2006. 


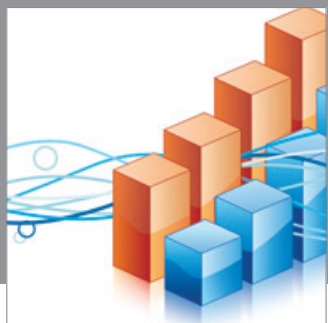

Advances in

Operations Research

mansans

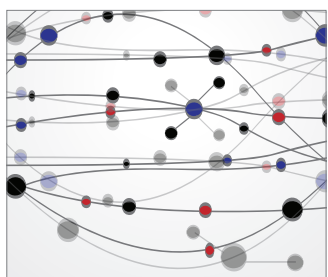

The Scientific World Journal
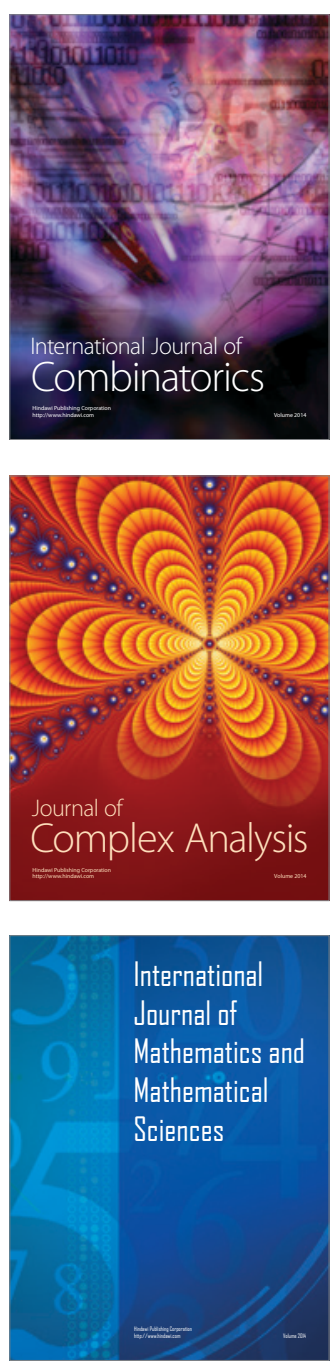
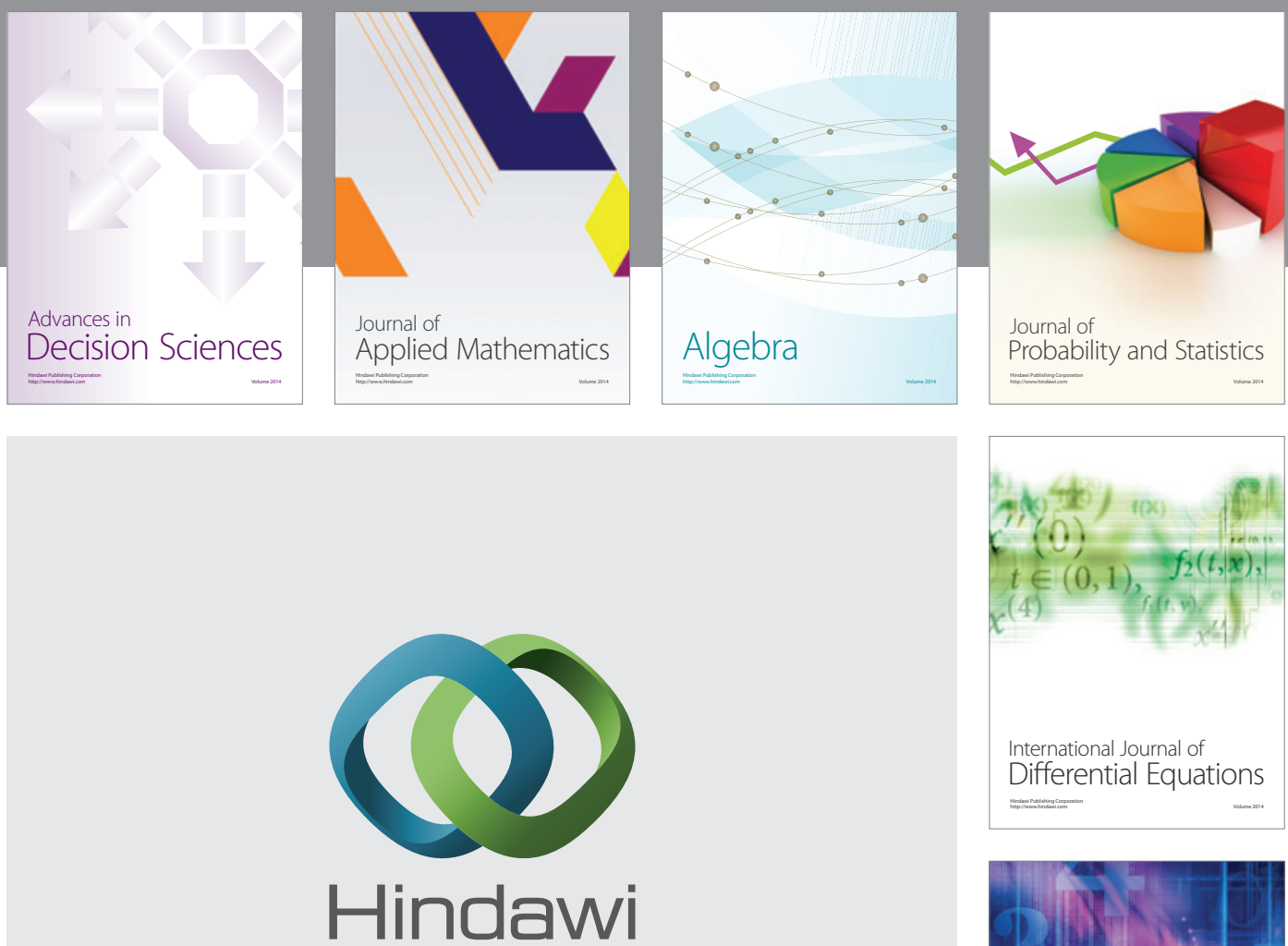

Submit your manuscripts at http://www.hindawi.com
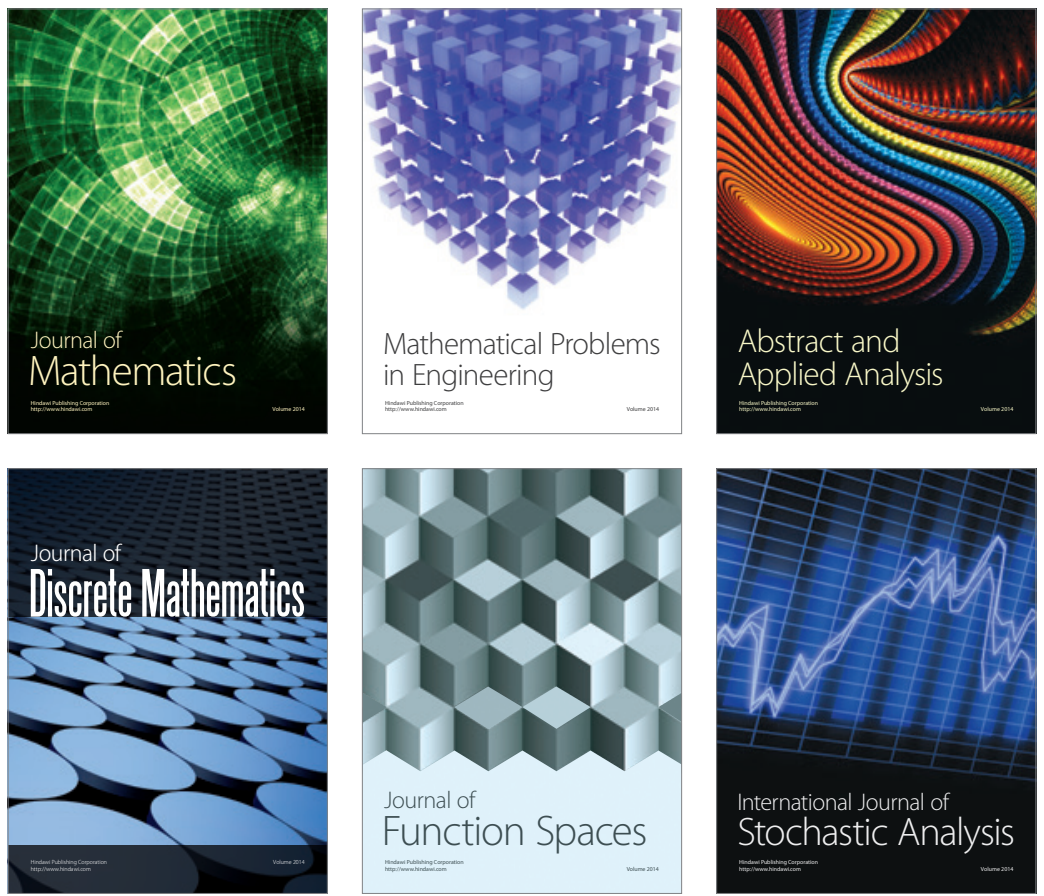

Journal of

Function Spaces

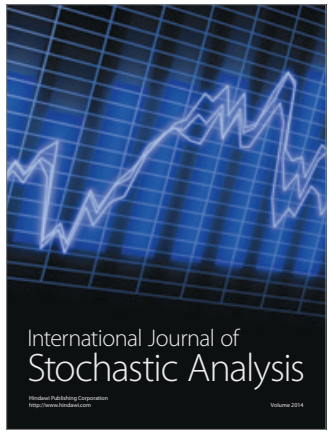

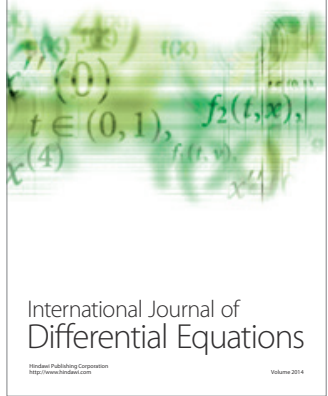
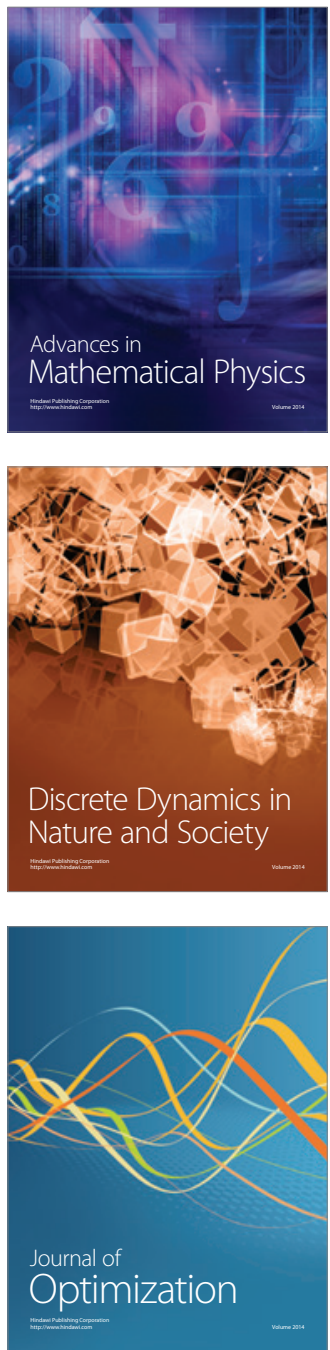